\title{
Beam Instability and Microbunching due to Coherent Synchrotron Radiation
}

\author{
S. Heifets and G. Stupakov \\ Stanford Linear Accelerator Center, Stanford University, Stanford, CA 94309
}

\section{INTRODUCTION}

A relativistic electron beam moving in a circular orbit in free space can radiate coherently if the wavelength of the synchrotron radiation exceeds the length of the bunch. In accelerators coherent radiation of the bunch is usually suppressed by the screening effect of the conducting walls of the vacuum chamber $[1,2]$. The screening effect is weak for short wavelengths, but if the wavelength is shorter than the length of the bunch, the coherent radiation becomes exponentially small. However, if the beam density has initially a fluctuation with a characteristic length much shorter than the bunch length, this fluctuation will radiate coherently. If the radiation results in the growth of the initial fluctuation one can expect micro-bunching of the beam and an instability which leads to increased coherent radiation at short wavelengths. Experimentally self-excited coherent microwave radiation has been observed recently in NSLS VUV ring at BNL [3, 4], at the Synchrotron Ultraviolet Radiation Facility (SURF) at NIST [5], and at the Advanced Light Source at LBL [6].

A beam microwave instability induced by the coherent radiation of the density fluctuations is studied theoretically in this paper. We consider the case when CSR of a bunch as a whole is suppressed by screening and is caused by microstructures with longitudinal dimensions small compared to the bunch length. In this case, we can simplify consideration studying stability of a coasting beam with the line density equal to the local line density of the bunch.

\section{EFFECT OF CSR ON COASTING-BEAM STABILITY}

Consider a coasting beam with the total number of particles $N$ and the relativistic factor $\gamma$ moving in a circular orbit of radius $R$ in free space. Let us use $(p, z)$ variables, where $p=\delta / \delta_{0}$ is the relative energy offset $\delta=\Delta E / E$ of a particle in units of the rms relative energy spread $\delta_{0}=\left\langle\delta^{2}\right\rangle^{1 / 2}, z$ is the longitudinal coordinate measured relative to the reference particle with the nominal energy, and $s=c t$.

The beam is described by the longitudinal distribution function $\rho(p, z, s)$ normalized so that $\int \rho(p, z, s) d z d p=$ $N$, where $N$ is the number of particles in the beam. This function satisfies the Vlasov equation

$$
\begin{gathered}
\frac{\partial \rho}{\partial s}-\eta \delta_{0} p \frac{\partial \rho}{\partial z} \\
-\frac{r_{0}}{\gamma \delta_{0}} \frac{\partial \rho}{\partial p} \int_{-\infty}^{\infty} d z^{\prime} d p^{\prime} W\left(z-z^{\prime}\right) \rho\left(p^{\prime}, z^{\prime}, s\right)=0
\end{gathered}
$$

where $\eta$ is the momentum compaction factor, $r_{0}$ is the classical electron radius, and the wake function $W\left(z-z^{\prime}\right)$ (per unit length of the path) describes the interaction with the coherent synchrotron radiation. In this equation we omitted the right-hand side that is responsible for the effects of the radiation damping. This approximation is valid if the growth rate of the instability is much larger than the inverse synchrotron radiation damping time.

Neglecting the screening effect of conducting walls we can write the wake function per unit length as a differential operator $[7,8]$

$$
W(\zeta)=-\frac{2}{\left(3 R^{2}\right)^{1 / 3} \zeta^{1 / 3}} \frac{\partial}{\partial \zeta} \text { for } \zeta>0,
$$

and $W(\zeta)=0$ for $\zeta \leq 0$. Note that unlike the traditional wake the radiation wakefield is localized in front of the moving charge.

We represent the distribution function $\rho$ as a sum of the equilibrium distribution function $\rho_{0}$ and a perturbation $\rho_{1}$

$$
\rho=\rho_{0}(p)+\rho_{1}(s, p, z),
$$

with $\rho_{1} \ll \rho_{0}$. Note that the equilibrium beam density $n_{b}$ is equal to $n_{b}=\int \rho_{0}(p) d p$, and the density perturbation $n_{1}$ is given by $n_{1}=\int \rho_{1}(p) d p$. Linearizing Eq. (1) and assuming that $\rho_{1}=\hat{\rho}_{1} e^{-i \omega s / c+i k z}$, where $k$ is the wavenumber, we find

$$
\left(\omega+c k \eta \delta_{0} p\right) \hat{\rho}_{1}=-i \frac{r_{0} c}{\gamma \delta_{0}} \frac{\partial \rho_{0}}{\partial p} Z(k) \int d p \hat{\rho}_{1}(p),
$$

where

$$
\begin{aligned}
Z(k) & =\int_{0}^{\infty} d \zeta W(\zeta) e^{-i k \zeta} \\
& =-\frac{2 i k^{1 / 3}}{3^{1 / 3} R^{2 / 3}} \int_{0}^{\infty} d \xi \xi^{-1 / 3} e^{-i \xi}=i A \frac{k^{1 / 3}}{R^{2 / 3}}
\end{aligned}
$$

The complex factor $A$ is $A=3^{-1 / 3} \Gamma\left(\frac{2}{3}\right)(\sqrt{3} i-1)=$ $1.63 i-0.94$, where $\Gamma$ is the gamma-function.

The non-trivial solution

$$
\hat{\rho}_{1}=-\frac{i c r_{0} Z(k)}{\gamma \delta_{0}\left(\omega+c k \eta \delta_{0} p\right)} \frac{d \rho_{0}}{d p} \hat{n}_{1}
$$

of Eq. (4) exists if $\omega$ satisfies the dispersion relation

$$
1=-\frac{i r_{0} c Z(k)}{\gamma \delta_{0}} \int \frac{d p\left(d \rho_{0} / d p\right)}{\omega+c k \eta \delta_{0} p},
$$

where $\hat{n}_{1}$ in Eq. (6) is the amplitude of the density perturbation, $\hat{n}_{1}=\int d p \hat{\rho}_{1}$. For the Gaussian distribution function, $\rho_{0}=n_{b}(2 \pi)^{-1 / 2} \exp \left(-p^{2} / 2\right)$, we can write Eq. (7) in the following form

$$
\frac{(k R)^{2 / 3}}{\lambda}=-\frac{A}{\sqrt{2 \pi}} \int_{-\infty}^{\infty} \frac{d p p e^{-p^{2} / 2}}{\Omega+p}
$$


where $\Omega=\omega / c k \eta \delta_{0}$,

$$
\lambda=\frac{n_{b} r_{0}}{\eta \gamma \delta_{0}^{2}}
$$

and $n_{b}$ is the number of particles in the beam per unit length. As always in stability theory, the integral on the right-hand side of Eq. (8) defines the dispersion function in the upper half-plane of the complex variable $\Omega$; the values of this function for $\operatorname{Im} \Omega<0$ are obtained by analytical continuation of the integral into the lower half-plane.

The plot of the function $\Omega$ versus $(k R)^{2 / 3} / \lambda$ obtained by numerical solution of Eq. (8) is shown in Fig. 1. The

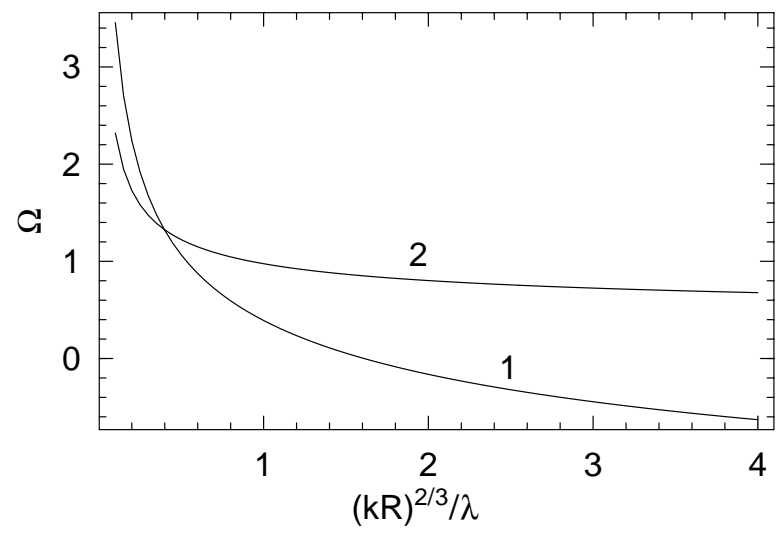

Figure 1: The imaginary ( $I$ ) and real (2) parts of $\Omega$ as functions of $(k R)^{2 / 3} / \lambda$.

imaginary part of the frequency is positive if $(k R)^{2 / 3} / \lambda<$ 1.6 which means the beam is unstable for such wavelength that

$$
k R<2.0 \lambda^{3 / 2} .
$$

Note that the condition for the instability is easier to satisfy for a beam with a small energy spread, since $\lambda \propto 1 / \delta_{0}^{2}$. This condition does not take into account the finite bunch length and the screening effect of the vacuum chamber walls which we discuss in the next section.

\section{EFFECT OF SCREENING AND FINITE BUNCH LENGTH}

For a bunched beam of length $\sigma_{z}$ and bunch population $N$ our results obtained in the coasting-beam approximation can be applied if the reduced wavelength of the modulation $1 / k$ is much smaller than the bunch length, $k \sigma_{z} \gg 1$. In this case the instability is controlled by the local value of the linear particle density $n_{b}$ with characteristic value of $n_{b}$ being $\sim N / \sigma_{z}$. Since $k$ is limited from above by the requirement (10), the micro-bunching instability can develop if the bunch length is large enough

$$
\sigma_{z} \gtrsim 0.5 \frac{R}{\lambda^{3 / 2}} .
$$

Another limitation to the theory is introduced by a finite aperture $b$ of the beam pipe. Assuming a pipe with a perfect conductivity, the coherent synchrotron radiation is suppressed due to the shielding effect at low harmonics with $k R \lesssim(\pi R / 2 b)^{3 / 2}$ [9]. Hence the instability can only develop for such values of $k$ that $2.0 \lambda^{3 / 2}>k R \gtrsim$ $(\pi R / 2 b)^{3 / 2}$. The requirement that the lower limit for the parameter $k R$ is less than the upper one gives the following necessary condition for the instability:

$$
\frac{R}{b} \lesssim \lambda
$$

If this inequality is not satisfied, finite energy spread in the beam suppresses the small-scale instability where the effect of the screening is not essential.

The above equations were derived for a circular orbit of the beam where $R=$ const. If this is not the case, one has to average the wakefield over the beam orbit which results in an additional factor $R /\langle R\rangle$ in Eq. (2), where $R$ is the bending radius of the magnets, and $\langle R\rangle=C / 2 \pi$ is the average radius of the beam orbit of circumference $C$. With this modification, instead of Eq. (9) one should use the following expression for the parameter $\lambda$ :

$$
\lambda=\frac{n_{b} r_{0} R}{\eta \gamma \delta_{0}^{2}\langle R\rangle}
$$

in Eqs. (11) and (12).

Finally, one has to take into account the effect of the incoherent synchrotron damping in the ring, which was neglected in Eq. (1). As a crude estimate of this effect, we may assume that the instability develops if the imaginary part of the frequency calculated above exceeds the synchrotron damping time $\gamma_{d}$. The effective growth rate of the instability in this case will be given by the difference $\operatorname{Im} \omega_{n}-\gamma_{d}$. A detailed study of the effect of the damping time on the instability threshold is given elsewhere [10].

\section{NONLINEAR REGIME OF INSTABILITY}

The above results are valid for the initial linear regime of the instability, when the amplitude of the perturbations is small. In the unstable regime, the growth of the initial perturbation will eventually evolve into nonlinear regime where the linear model breaks down. One can expect a saturation of the instability at some level in nonlinear regime. We will estimate here the amplitude of the instability at the saturation using simple order of magnitude arguments; a more detailed study of the nonlinear regime is published elsewhere [11].

Let us assume that the perturbation of the beam density can be written as $\hat{n}_{1} e^{-i \omega s / c+i k z}$. The wake that is generated by this perturbation is

$$
\begin{aligned}
w_{1} & =\int_{-\infty}^{\infty} d z^{\prime} W\left(z-z^{\prime}\right) n_{1}\left(z^{\prime}, s\right) \\
& =i A \hat{n}_{1} \frac{k^{1 / 3}}{R^{2 / 3}} e^{-i \omega s / c+i k z}
\end{aligned}
$$


The particles in the beam move under the influence of this wake according to the equation of motion

$$
\delta_{0} \frac{d p}{d s}=-\frac{r_{0}}{\gamma} w_{1}
$$

which solution is

$$
p=\frac{A \hat{n}_{1} r_{0} c k^{1 / 3}}{\gamma \delta_{0} R^{2 / 3} \omega} e^{-i \omega s / c+i k z} .
$$

When the amplitude of the oscillations becomes comparable with width of the distribution function, $\Delta p \sim 1$, the linear theory becomes invalid, and one can expect a saturation of the instability. This gives an order of magnitude estimate for the maximum density perturbation

$$
\hat{n}_{1} \sim \frac{\gamma \delta_{0}^{2} \eta(k R)^{2 / 3}}{r_{0}}
$$

where we have used the relation $\omega \sim c k \eta \delta_{0}$. Of course, this formula is valid if the density perturbation is smaller than the equilibrium beam density, $\hat{n}_{1} \ll n_{b}$, which is satisfied above the threshold due to Eq. (10).

\section{NUMERICAL ESTIMATES}

We estimated conditions for the instability in the Low Energy Ring (LER) of PEP-II accelerator at SLAC, Advanced Light Source at the Berkeley National Laboratory, and the VUV ring at the National Synchrotron Light Source at BNL.

The machine parameters and the calculated values of $\lambda, R / 2 \lambda^{3 / 2}$, and the ratio $R / b$ are presented in Table 1 . For LER the parameter $\lambda$ is smaller than the ratio $R / b$ so that the condition (12) is not satisfied. For both ALS and VUV rings the condition (12) is satisfied. The parameter $R / 2 \lambda^{3 / 2}$ which should be compared with the bunch length [see Eq. (11)] is smaller than $\sigma_{z}$ for ALS and VUV rings. Hence, according to the theory, the CSR instability is possible in ALS and VUV rings, which may explain recent observations of bursts of coherent radiation in the experiments $[3,4,6]$.

\section{CONCLUSION}

For a given beam pipe aperture, coherent synchrotron radiation of a bunch as a whole is possible only for relatively short bunches. We showed that CSR itself can produce such a bunch modulation provided that linear bunch density is above threshold given by the criterion Eq. (10). In this case the bunch becomes unstable due to the CSR impedance in a range of wavenumbers $k$. Modes with large values of $k$ are stabilized by the energy spread in the beam because $Z(k) / k$ rolls off as $k^{-2 / 3}$. Modes with low $k$ are usually stable because CSR impedance is suppressed by the wall screening effect. It is worthwhile to notice that the threshold depends on the rms energy spread $\delta_{0}$. Criterion Eq. (10) shows that cooling of a bunch with given bunch
Table 1: Beam and instability parameters for PEP-II LER, ALS and VUV NSLS rings.

\begin{tabular}{|l|l|l|l|}
\hline \hline Accelerator & PEP-II LER & ALS & VUV \\
\hline$E(\mathrm{GeV})$ & 3.1 & 1.5 & 0.81 \\
\hline$\eta$ & $1.31 \cdot 10^{-3}$ & $1.41 \cdot 10^{-3}$ & $2.35 \cdot 10^{-2}$ \\
\hline$\delta_{0}$ & $8.1 \cdot 10^{-4}$ & $7.1 \cdot 10^{-4}$ & $5.0 \cdot 10^{-4}$ \\
\hline$\langle R\rangle(\mathrm{m})$ & 350 & 31.3 & 8.11 \\
\hline$R(\mathrm{~m})$ & 13.7 & 4 & 1.91 \\
\hline$b(\mathrm{~cm})$ & 2 & 1 & 2.1 \\
\hline$I_{b}(\mathrm{~mA})$ & 2 & 30 & 400 \\
\hline$\sigma_{z}(\mathrm{~cm})$ & 1 & 0.7 & 4.7 \\
\hline$\lambda$ & 7 & 1200 & 250 \\
\hline$R / b$ & 550 & 400 & 90 \\
\hline$\frac{R}{2 \lambda^{3 / 2}}(\mathrm{~cm})$ & 1.0 & 0.005 & 0.025 \\
\hline
\end{tabular}

population always make bunch unstable and might lead to micro-bunching of the beam. Dynamics of the modulation above the threshold is complicated and may lead to steady state modulation. Eq. (17) gives the estimate of the equilibrium amplitude of modulation set by the reverse effect of the growing mode on the distribution function and their interaction.

\section{ACKNOWLEDGEMENTS}

We thank R. Warnock for pointing out recent publications on experimental observations of coherent synchrotron radiation.

This work was supported by Department of Energy contract DE-AC03-76SF00515.

\section{REFERENCES}

[1] J. Schwinger, unpublished, (1945). See Report LBL-39088, (1996); L. Schiff, Rev. Sci. Instr. 17, 6 (1946).

[2] R. L. Warnock and P. Morton, Part. Accel. 25, 113 (1990).

[3] J. B. Murphy, Talk presented at the Workshop on Broadband Impedance Measurements and Modeling, SLAC, Stanford (February 2000).

[4] G. L. Carr et al. , NIM (2001), to be published.

[5] U. Arp et al., Phys. Rev. PRST-AB 4, 054402 (2001).

[6] J. Byrd, private communication.

[7] J. B. Murphy, S. Krinsky and R. L. Gluckstern, PAC 1995, p.2980, (IEEE, Piscataway, NJ, 1996).

[8] Y. S. Derbenev et al., DESY FEL Report TESLA-FEL 9505, (September 1995).

[9] R. L. Warnock, Report SLAC-PUB-5375, SLAC (1990).

[10] S. Heifets and G. Stupakov, Report SLAC-PUB-8803, (March 2001).

[11] S. Heifets and G. Stupakov, Report SLAC-PUB-8758, (January 2001). 